\title{
Physical Stress Echocardiography Evaluation in Athletes with Bicuspid Aortic Valves
}

\author{
Laura Stefani, Roberto Mercuri, Gabriele Innocenti, Loira Toncelli, Maria Concetta Robertina Vono, \\ Giorgio Galanti
}

Department of Sports Medicine Centre, Non-Invasive Cardiac Laboratory, University of Florence, Florence, Italy.

Email: laura.stefani@unifi.it

Received August $9^{\text {th }}, 2010$; revised August 10 $0^{\text {th }}, 2010$; accepted August $12^{\text {th }}, 2010$.

\begin{abstract}
Objective: Bicuspid Aortic Valve (BAV) is normally asymptomatic for a long time in both the general population and regularly-trained athletes. The study aimed to evaluate the role of physical stress echocardiography in early identification of any possible dissimilarities in the Left Ventricle $(L V)$ performance and the valve functioning of asymptomatic BAV athletes as compared to the Tricuspid Aortic Valve (TAV) athletes. Design: Data were collected for BAV and TAV athletes from echocardographic examinations to evaluate any possible differences between them. Setting: Sport Medicine Center - University of Florence-Italy. Participants: 66 male BAV athletes and 45 TAV athletes Assessment of risk factors: decrease in LV performance and aortic valve dysfunction during stress test. Main outcome measures: Measure of the standard echocardiographic parameters. Comparison of LV Ejection Fraction (EF) and Aortic Peak Flow Velocity (APFV) data in the two groups at rest and after stress. Results: At rest, values were normal in both groups, although $L V$ systolic diameters tended to be higher in BAV. After physical effort, APFV and EF showed a significant increase in both groups, and for the former the values were at the upper limits in $B A V\left(2.55 \mathrm{~m} / \mathrm{s}^{B A V} 2.12 \mathrm{~m} / \mathrm{s}^{T A V}\right)$. Conclusions: The data confirm normal LV performance in asymptomatic BAV athletes. Considering the tendency of this group to show slight enhancement of both APFV and LV dimensions in this group periodical evaluation with physical stress echography might be proposed as a follow-up procedure for early detection of any additional effect of physical exercise.
\end{abstract}

Keywords: Bicuspid Aortic Valve, Physical Stress Echography, Athletes

\section{Introduction}

Bicuspid Aortic Valve (BAV) is one of the most common congenital cardiac conditions in both the general population [1] and athletes [2], and it generally remains asymptomatic for a long time. BAV is commonly considered a disease of the aortic tract; in fact it is associated with an enlargement of the proximal ascending aortic vessel [3]. Although in adults aortic valve dysfunction tends to develop into stenosis, the most frequent Doppler pattern found in young athletes is insufficiency of the aortic valve [2-4]. Recently several authors have hypothesized that BAV may affect the contractile properties of the entire myocardium $[5,6]$. Defects in the genes that encode matrix elements have not yet been identified in patients with BAV. Although the gene for fibrillin-1 may be structurally normal, transcriptional elements that control protein production may be defective [1]. The hypothesis that a parallelism exits between the BAV disease and the Marfan syndrome as regards fibrillin-system deficiency is therefore a very interesting one. This hypothesis is supported by the progressive enlargement of the Left Ventricle (LV) chamber recently demonstrated in a five-year follow-up study conducted on a group of BAV athletes affected with mild aortic insufficiency, where the extent of the valve dysfunction cannot justify the LV chamber increase [4].

Physical stress echocardiography is one of the most frequent hemodynamic approaches in evaluating the performance of both myocardium and valves. In the case of BAV with aortic valve regurgitation, this test allows assessment of various measures of myocardial and valvular function during an acute exercise bout, with high diagnostic accuracy, sensitivity and reproducibility [7]. In athletes, stress echocardiography is an ideal non-invasive tool for estimating the impact of physical training on cardiac function at rest and during exertion.

Using physical stress echocardiography, we investigated overall performance of the left ventricle (LV) and valve function in a group of BAV athletes with mild aor- 
tic regurgitation, versus athletes with normal Tricuspid Aortic Valve (TAV). The aim of this study was to assess whether BAV was associated with early myocardial dysfunction in athletes. The hypothesis was that physicalstress echography might add important information on the heart performance of athletes who have BAV with associated mild aortic dysfunction and slight enlargement of the LV chamber, despite their not yet being symptomatic.

\section{Materials}

\subsection{Subjects}

All the subjects enrolled in the study were informed about the methodology and the aims of the study and gave their informed consent to participate in this protocol, which was approved by the Ethics Committee of our institution.

In the period from January 1999 to December 2006, a group of 66 BAV athletes (58 males and 8 females; average age $25 \pm 11$ years) were identified during the regular pre-participation screening among a large cohort of subjects examined. All these athletes have never discontinued their sport activity during this period, they were regularly trained and yearly followed at Sports Medicine Center in Florence-Italy by a complete standard echocardiographic exam at rest and by physical echo-stress, conducted up to the maximal effort.

The inclusion criteria to be enrolled in the protocol study were the absence of aortic valve stenosis, or a severe aortic valve insufficiency or a pathologic enlargement of ascending aortic tract demonstrated at the echocardiographyc exam in addition to the evidence of clinical symptoms. All the BAV subjects studied were in fact asymptomatic and had mild aortic insufficiency $(+1$ or +2 ) evaluated by color flow examination according to the ACC/AHA Guidelines [8].

The BAV group was substantially homogeneous respect of the dynamic and static component of the sports practiced [9]. Among the BAV athletes in 43 soccer players, 8 swimmers, 7 basketball players, 4 rugby players and 1 water polo player (63 athletes), the dynamic component of the workload was of high-moderate level associated with a low-moderate static component and only in two cases (2 rowers), both components were high, while for the judo player the static component was predominant.

The athletes were regularly trained for two hours at $80 \%$ of their $\mathrm{VO}_{2}$ max, three times a week for at least 8 months per year. The BAV athletes were matched with 45 healthy TAV athletes (20 soccer players, 10 swimmers, 8 rugby players, and 7 basketball players) practicing sports with a similar dynamic component and lowmoderate static workload.

Before to start with the echocardiographic exam, the
BAV and TAV athletes were both submitted to the same clinical evaluation, (previous history and general examination), to exclude the presence of cardiovascular or metabolic diseases.

\section{Methods}

BAV and TAV athletes were submitted to a complete echocardiographic exam at rest with calculation of the standard systolic and diastolic myocardial parameters, to confirm from the short-axis view, the presence of bicuspid or tricuspid valve. During the physical stress the behaviors of the aortic valve and of the left ventricle function were estimated by the measurement of the dynamic echo-parameters.

\subsection{Echocardiographic Exam}

The echocardiographic study was performed by two experienced board-certified cardiologists, using a My Lab 50 echocardiograph (Esaote-Italy) equipped with a $2.5 \mathrm{MHz}$ probe. The two cardiologists had worked together for more than 5 years, and no formal reliability verification of the studies (inter- or intra-tester) was performed.

From the evaluation of the valve morphology and of the position of the cusp fusion, the BAV valves were classified as typical R-L (right-left coronary cusp fusion) or atypical R-N (right-non-coronary cusp fusion) [10].

In accordance with AHA Guidelines [11], the following basal two-dimensional systolic and diastolic and Doppler echocardiographic parameters at rest were calculated: interventricular septum (IVS) and posterior wall (PW) thickness, left ventricular end diastolic diameter (LVEDd), left ventricular end systolic diameter (LVESd), left atrium dimensions (LAD) and aortic root (Aor) dimensions, peak velocities of pulsed wave Doppler transmitral flow during early diastole (E) and atrial systole (A), deceleration time (DT) of early diastolic flow, and isovolumic relaxation time (IVRT). Analysis of the diastolic parameters was performed in the presence of a stable R-R interval from a series of three measurements. Evaluation of the left ventricular cardiac mass index $\left(\mathrm{CMI} \mathrm{g} / \mathrm{m}^{2}\right)$ was obtained using the Devereux procedure [12], and the ejection fraction (EF) (\%) was calculated from the formula [(LV end-diastolic3 - LV end-systolic3) /LV end-diastolic3] × $100(\%)$. LV Relative Wall Thickness (RWT) was calculated as the ratio of the posterior and septum wall thickness to end-diastolic diameter, according to the formula: $[2 \times(\mathrm{PWTd}+\mathrm{IVSd}) / \mathrm{LVDd}]$. A RWT value of 0.40 was considered as the cut-off point [13].

The diameter of the aortic root was performed in MMode from the long-axis view.

From the five-chamber view, peak systolic aortic velocity and the degree of valve insufficiency were determined by continuous wave and color Doppler analysis. In accordance with the ACC/AHA Guidelines [11], the se- 
verity of valve insufficiency was described as the extent of the regurgitant flow on a 0 to $4+$ scale, using the colorflow mapping method. At rest, three clips, including a series of three heart cycles of LV chamber 2D images, two in short-axis view at the mitral valve and apical plane (Figure 1, Figure 2) and one in four-chamber view (Figure 3), were captured for the basic evaluation of the myocardial performance.

\subsection{Physical Stress Echocardiography}

The stress test was performed using the Bruce protocol on a commercially-available cycloergometer with strikeback (Ergoline by Esaote-Italy), using 25-watt increments every two minutes. A symptom-limited test maximal exercise test was undertaken. The athletes cycled supine, with their chest on a strike-back. At the peak of the exercise test, Aortic Peak Flow Velocity (APFV) was estimated by placing the Doppler cursor just beyond the aortic valve in the apical five-chamber view. The degree of

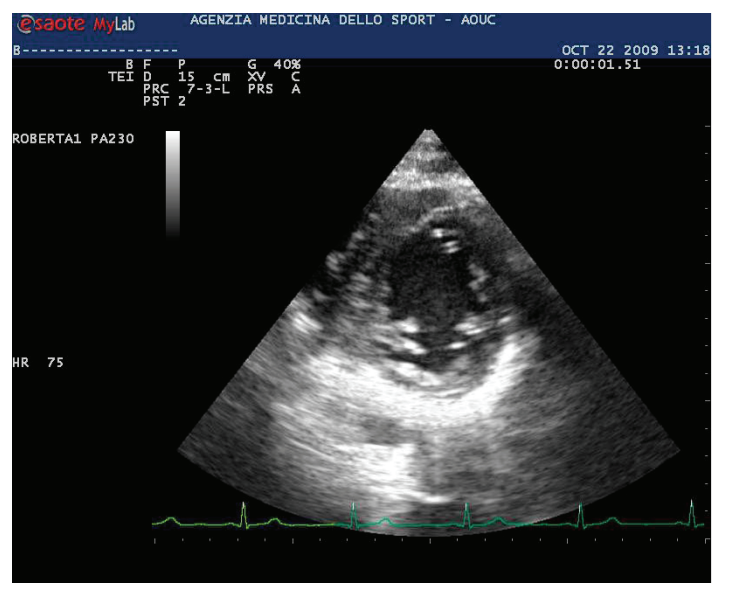

Figure 1. The 2D short axis picture shows the segments of the $\mathrm{LV}$ chamber at mitral valve plane in rest condition

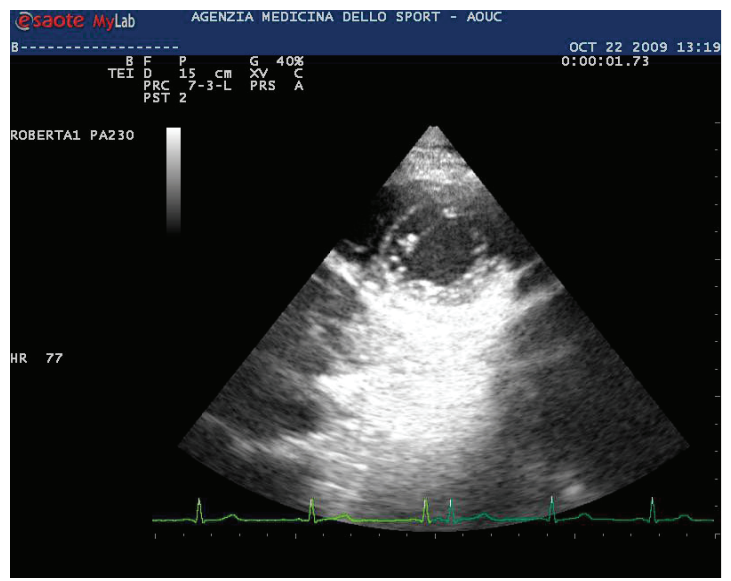

Figure 2. Short axis 2D image of the myocardial segments of the $L V$ chamber at the apical level in rest condition

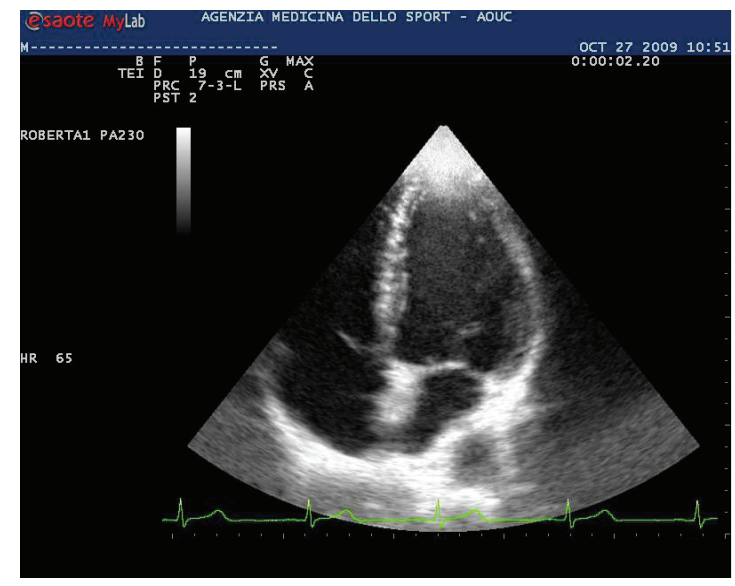

Figure 3. The 2D picture in four chamber view shows the LV chamber at rest

residual aortic regurgitation at the end of the stress was estimated with the Doppler and colour-scale analysis from the five-chamber view. Since high heart and respiratory rates make both Simpson biplane images particularly difficult to obtain at peak exertion, EF was measured from the long-axis view as the difference between the LVEDd and LVESd/LVEDd $\times 100$ in M-mode corresponding to the shortening fraction. This is an easy and accurate method for estimating EF [14]. It is currently used when the LV chamber is geometrically regular and free of any significant valve regurgitation, as in this context.

Wall motion abnormalities were evaluated by comparing LV chamber contractility at the beginning and at the end of maximal exertion. For this purpose two 2D echo clips, including a series of three heart cycle images, obtained in short-axis view at mitral valve plane (Figure 4) at the apical level (Figure 5), and one other clip in fourchamber view (Figure 6), were captured and compared to those previously obtained at rest.

\subsection{Statistical Analysis}

Statistical analysis was performed using the SPSS 13.0 package for Windows XP. All data are expressed as mean \pm Standard Deviation. The groups were compared using Student's unpaired $t$-test, while Student's paired $t$-test was used to compare the results within each group. A probability value (p) of $<0.05$ was considered statistically significant.

\section{Results}

\subsection{Echocardiographic Findings at Rest}

The two groups did not show any significant differences for age, systolic blood pressure (SBP), diastolic blood pressure (DBP), heart rate ( HR) or body surface area (BSA) (Table 1). 


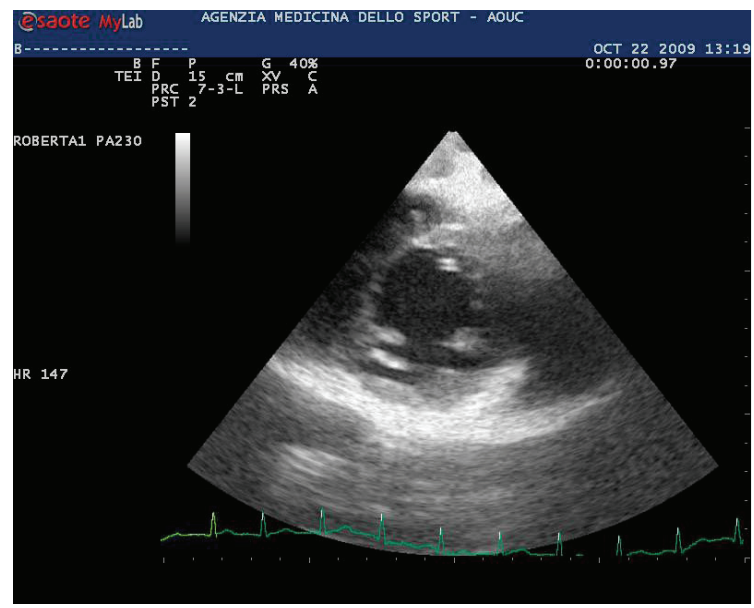

Figure 4. 2D short axis image of the LV chamber segments at the mitral plane during stress

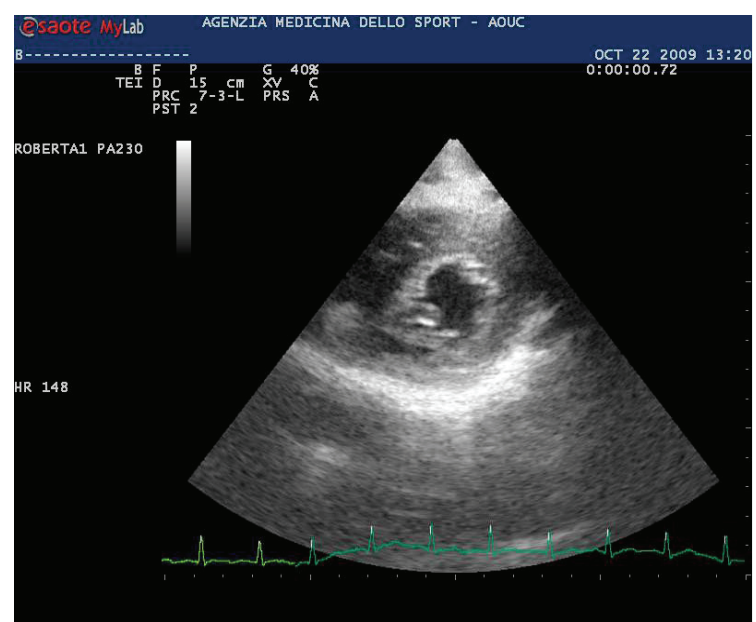

Figure 5. 2D short axis picture of the LV apical myocardial segments during stress

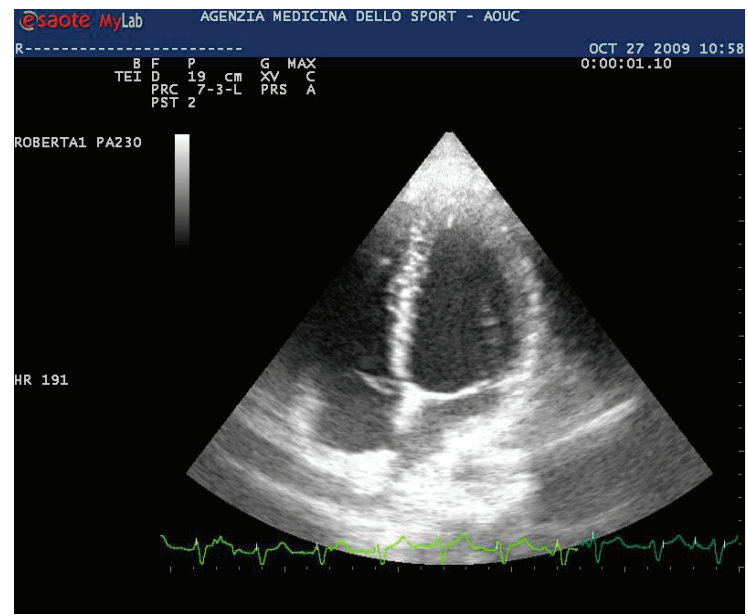

Figure 6. The 2D picture in four chamber view shows the LV chamber segments at the maximal effort
Of the 66 BAV athletes studied, 52 had fusion of the R-L cups (typical), and in 14 the fusion was atypical (R$\mathrm{N})$. At rest, the standard LV systolic and diastolic echocardiographic parameters were within the normal range (Table 1) in both groups. Some differences between BAV and TAV were present regarding the LVESd (31.69 $\pm 4.02 \mathrm{~mm}^{\mathrm{BAV}}$ vs $\left.29.3 \pm 2.8^{\mathrm{TAV}} \mathrm{mm} ; \mathrm{p}<0.01\right)$. Two measures of the diastolic function: IVRT $\left(76.36 \pm 8.64 \mathrm{~ms}{ }^{\mathrm{BAV}}\right.$ vs. $\left.66.85 \pm 6.45^{\mathrm{TAV}} \mathrm{ms} ; \mathrm{p}<0.01\right)$ and DT $(184.69 \pm$ $25.32 \mathrm{~ms} \mathrm{vs}^{\mathrm{BAV}} 171 \pm 25.37 \mathrm{~ms}^{\mathrm{TAV}}$; $\mathrm{p}<0.001$; Table 1) were higher in BAV than in TAV athletes. Mean RWT values were lower than 0.40 for all athletes; CMI values were within the normal range in both BAV and TAV athletes, with no evidence of $\mathrm{LV}$ concentric or eccentric remodelling.

EF values were within normality in both groups, despite a slight tendency to lower values in BAV as opposed to TAV athletes.

Aortic root diameters showed no significant differences in either group; consequently aortic root pathology has not been documented in our data.

Table 1. General and echocardiographic parameters at rest

\begin{tabular}{cccc}
\hline & 66 BAV athletes & 45 TAV athletes & p \\
\hline Age (years) & $25.4 \pm 1$ & $22.77 \pm 0.91$ & NS \\
SBP $(\mathrm{mmHg})$ & $114.8 \pm 10.64$ & $110 \pm 4$ & NS \\
DBP $(\mathrm{mmHg})$ & $73.58 \pm 7.81$ & $74.23 \pm 5.60$ & NS \\
HR & $62.22 \pm 5.40$ & $60.19 \pm 4.50$ & NS \\
BSA $\left(\mathrm{m}^{2}\right)$ & $1.83 \pm 0.25$ & $1.79 \pm 0.15$ & NS \\
LAD $(\mathrm{mm})$ & $32.96 \pm 3.70$ & $31.38 \pm 2.87$ & NS \\
IVS $(\mathrm{mm})$ & $9.81 \pm 0.91$ & $9.92 \pm 0.97$ & NS \\
PWT $(\mathrm{mm})$ & $9.50 \pm 0.99$ & $9.31 \pm 0.88$ & NS \\
Aor $(\mathrm{mm})$ & $30.81 \pm 4.01$ & $29.85 \pm 2.23$ & NS \\
RV $(\mathrm{mm})$ & $20.18 \pm 4.06$ & $19.68 \pm 2.06$ & NS \\
LVEDD $(\mathrm{mm})$ & $50.65 \pm 4.45 \mathrm{~mm}$ & $48.6 \pm 2.5 \mathrm{~mm}$ & NS \\
LVESD $(\mathrm{mm})$ & $31.69 \pm 4.02 \mathrm{~mm}$ & $29.3 \pm 2.8 \mathrm{~mm}$ & $\mathrm{p}<0.01$ \\
DT $(\mathrm{ms})$ & $184.69 \pm 25.32$ & $171 \pm 25.37$ & $\mathrm{p}<0.05$ \\
IVRT $(\mathrm{ms})$ & $76.36 \pm 8.64$ & $66.85 \pm 6.45$ & $\mathrm{p}<0.01$ \\
E peak $(\mathrm{cm} / \mathrm{s})$ & $82.84 \pm 18.44$ & $91.85 \pm 22.05$ & NS \\
A peak $(\mathrm{cm} / \mathrm{s})$ & $47.87 \pm 13.03$ & $50.31 \pm 10.76$ & NS \\
CMI $\left(\mathrm{g} / \mathrm{m}^{2}\right)$ & $115.45 \pm 19.85$ & $109.30 \pm 15.71$ & NS \\
EF $(\%)$ & $68.8 \pm 2.3$ & $69.2 \pm 5.7$ & $\mathrm{p}<0.05$ \\
A P FV rest $(\mathrm{m} / \mathrm{s})$ & $1.70 \pm 0.34$ & $1.73 \pm 0.10$ & NS \\
RWT $(\%)$ & $0.37 \pm 0.026$ & $0.38 \pm 0.002$ & NS \\
\hline
\end{tabular}

SBP: Systolic Blood Pressure; DBP: Diastolic Blood Pressure; HR: Heart Rate; BSA: Body Surface Area; LAD: Left Atria Dimension; IVS: Inter Ventricular Septum; LW: Lateral Wall; Aor: Aortic root dimension; RV: Right Ventricle; LVEDD: Left Ventricular End- Diastolic Dimension; LVESD: Left Ventricular End-Systolic Dimension: DT: Deceleration Time; IVRT: Isovolumic Relaxation Time; E peak: E (velocity) Peak; A peak: A (velocity) Peak; CMI: Cardiac Mass Index; EF: Ejection Fraction; AP F V: Aortic Peak Flow Velocity; RWT: Relative Wall Thickness 
At echocardiographic color scale Doppler, $48 \mathrm{BAV}$ athletes showed mild aortic regurgitation $(+1$ grade), and grade +2 was found in the remaining 18 athletes. Aortic valve systolic flow peak velocity values found at the Doppler analysis were within the normal range in 46 of the BAV athletes studied. In 22 subjects, 19 males and 3 females, the aortic flow velocity values estimated from the five-chamber view by CW (Continuous Wave) Doppler analysis were at the upper limits (average values of $1.8 \pm$ $2 \mathrm{~m} / \mathrm{s}$ ). These data were in any case associated with a low gradient $(<20 \mathrm{mmHg})$, and therefore compatible with sport activity.

In the TAV group, the Doppler pattern of the aortic valve was normal.

\subsection{Physical Stress Echocardiography Results}

With the physical stress echocardiography examination EF, measured at the end of the effort test in M-mode from the long-axis view, showed a significant improvement in both parameters as compared to the resting state (EF\% $82.9 \pm$ $5.1^{\mathrm{TAV}} ; \mathrm{EF} \% 74.39 \pm 3.10^{\mathrm{BAV}}$ with $\left.\mathrm{p}<0.001\right)$. Similarly, the aortic flow velocity profile increased significantly in the two groups of athletes after stress as compared to the resting condition $\left(2.12 \pm 0.1 \mathrm{~m} / \mathrm{s}{ }^{\mathrm{TAV}}\right.$ vs. $2.55 \pm 0.48 \mathrm{~m} / \mathrm{s}{ }^{\mathrm{BAV}} \mathrm{p}$ $<0.001$ ) (Table 2). In the athletes as a whole, the degree of aortic regurgitation showed a physiological reduction from $2+$ to $1+$ level in 18 subjects, while for the other 48 athletes it disappeared completely. In both groups a physiological increase in the HR was recorded during effort. The average HR value, calculated at the end of the stress test, was $160 \pm 5$ for BAV and $162 \pm 3$ for TAV.

Wall motion was evaluated by comparing the $2 \mathrm{D}$ echo-images in short-axis view regarding the basal and apical segments of the LV chamber in rest and stress conditions. Two more images in four-chamber view were obtained to better compare all the myocardial segments. No abnormality was evident, hence normal LV performance during effort was confirmed in both groups.

\section{Discussion}

BAV is usually considered to be an aortic tract condition associated with progressive enlargement of the proximal ascending aorta. The latter aspect has generally been considered to be the main characteristic of this congenital cardiac disease, and to be closely linked to an increased risk of sudden death in subjects with BAV. The most fre- quent Doppler pattern found in the young (both athletes and general population) is aortic valve insufficiency [2-4], while progression to aortic valve stenosis is more frequently associated with the older population Few data are so far available on the possible mechanisms involved in the progression of valve dysfunction to insufficiency rather than to stenosis. Previous studies have identified the main reason for differing progression towards the two kinds of valve dysfunction as lying in the differing cusp fusion of the aortic valve [15]. Authors have recently highlighted in the BAV athlete population the possible impact of regular and competitive exercise on both the aortic valve and myocardial function. Normally-functioning BAV does not usually constitute a limit for practicing sport, even if it is known that the stress of regular and intense exercise on an abnormal aortic valve may indeed favor its early deterioration and accelerate the development of complications. Athletes with BAV thus require regular follow-up, which should include an echocardiographic assessment at least every year [16].

The presence of aortic valve dysfunction moreover increases the importance of evaluating EF, particularly during stress. EF is currently considered to be one of the best parameters for an accurate assessment of left ventricle function, both in the presence of aortic insufficiency (often associated with hyperdynamic LV function) and in presence of valve stenosis, where the complex relationship between the area of the aortic valve and LV function requires estimation of the contractile reserve [17] of the aortic valve. $\mathrm{EF}$ is also used for long-term monitoring to assess myocardial performance and to establish surgical timing for valve replacement $[18,19]$.

In the present investigation, the measure of valve contractile reserve has not been considered in any case due to the complete absence of subjects showing a significant aortic valve gradient.

Starting from this background, physical stress can therefore be considered to be a further helpful tool for discovering early signs of slight myocardial and valve failure in regularly-trained athletes who are not yet symptomatic [7] but are submitted to a daily workload.

Recently, several authors have also suggested possible involvement of the contractile property of the entire myocardium in BAV patients. Their hypothesis is that a different expression of the metalloproteinase system (MMPs) in BAV as compared to TAV subjects might indicate the presence of a pathological process which also involves the myocardium $[5,6]$.

Table 2. Echocardiographic and Doppler parameters during physical stress echocardiography

\begin{tabular}{ccccccc}
\hline & APFV rest & APFV stress & $\boldsymbol{P}$ & EF rest & EF stress & $\boldsymbol{P}$ \\
\hline BAV & $1.70 \pm 0.34 \mathrm{~m} / \mathrm{s}$ & $2.55 \pm 0.48 \mathrm{~m} / \mathrm{s}$ & $<\mathbf{0 . 0 0 1}$ & $68.87 \pm 3.4 \%$ & $74.39 \pm 3.10 \%$ & $<\mathbf{0 . 0 0 1}$ \\
TAV & $1.73 \pm 0.10 \mathrm{~m} / \mathrm{s}$ & $2.12 \pm 0.1 \mathrm{~m} / \mathrm{s}$ & $<\mathbf{0 . 0 0 1}$ & $69.2 \pm 5.7 \%$ & $82.9 \pm 5.1 \%$ & $<\mathbf{0 . 0 0 1}$ \\
\hline
\end{tabular}

APFV: Aortic Peak Flow Velocity; EF: Ejection Fraction 
In our BAV and TAV athletes, LV performance at rest was normal. There was however a slight but significant difference in the LVESd between the BAV and TAV groups.

On a first examination these results can be considered to agree with our previous data [4], where the BAV group showed normal dimensions of the LV chamber, despite a significant progressive enlargement as compared with TAV athletes.

Since the degree of valve insufficiency was mild, we cannot attribute this minor increase in LV dimensions and the modifications in the haemodynamic parameters to any impact of aortic valve dysfunction.

On the other hand, the different diastolic patterns found at rest in the two groups analyzed cannot yet be well interpreted from these preliminary data. The usefulness of the diastolic pattern as a marker of an initial metalloproteinase system failure can at present only be hypothesized. Further investigations regarding genetic and histological data will be of clinical interest.

The hemodynamic parameters obtained from the stress test showed a significant physiological increase in EF and in aortic flow velocity in all the athletes. The progressive enhancement of the EF confirmed that myocardial contractile response was normal for both groups, even if the response to physical exertion in TAV subjects seemed to be higher than in BAV ones, while still remaining in the normal range.

Actually we demonstrated a significant improvement of EF under stress as compared to rest conditions in both TAV and BAV groups. This particular result does not however allow us to conclude that there is a significant difference between BAV and TAV.

Moreover, it is interesting to note that the particular increase in AFPV values in BAV subjects (average 2.55 $\mathrm{m} / \mathrm{s}$ ) as compared with TAV ones (average $2.12 \mathrm{~m} / \mathrm{s}$ ) at the end of stress, could suggest early degeneration of the aortic valve towards stenosis.

Unfortunately, current literature does not report any precise indications of the AFPV cut-off values which might serve as a predictive pointer of exacerbation of this symptom.

The possible different behavior of this parameter in BAV athletes with typical cusp fusion as compared with those with an atypical one might be considered, but this comparison has not been included in the present investigation. A larger cohort of subjects would need to be studied to clarify this point.

This study in fact addresses a relatively select population of BAV subjects, enrolled from those normally followed at our regional Center. The study group did not include subjects who had discontinued their sports activity due to progressive aortic valve dysfunction, or subjects with BAV who had never practiced sports.

Some limits can be identified in the present study.
First of all, the investigation involves static evaluation of the haemodynamic parameters and does not include any analysis of data deriving from periodical echo-stress follow-up. Results from a long period of observation could help clarify the sensitivity of the stress-test in this particular group of athletes.

The progressive enlargement of the LV chamber previously described in them [4] does not in fact directly or immediately correspond to the onset of clinical symptoms. Comparison with a group of athletes having TAV associated with mild aortic regurgitation might be considered as the next step to better understanding of the readings near the upper limits of the normal range in the BAV group. In conclusion, it is not yet possible from these data to clearly identify any functional involvement of the myocardium in BAV, and further studies will be necessary for this particular aspect.

However, considering the progressive enlargement of the left ventricle [4] and the particular APFV increase in $\mathrm{BAV}$, it might be useful to propose a regular follow-up program with stress-echo for early detection of any involvement of the heart, including both LV myocardial performance and aortic valve functioning. For the latter, the progressive aortic-ventricular gradient must be closely monitored.

\section{Acknowledgements}

We thank Susan Charlton, Language Assistant at the University of Florence, for her English language revision.

\section{REFERENCES}

[1] P. W. M. Fedak, S. Verna and T. E. J. David, "Clinical and Pathophysiological Implications of Bicuspid Aortic Valve," Circulation, Vol. 106, No. 8, August 2002, pp. 900-904.

[2] L. Stefani, G. Galanti, L. Toncelli, et al., "Bicuspid Aortic Valve in Competitive Athletes," British Journal of Sports Medicine, Vol. 42, No. 1, January 2008, pp. 31-35.

[3] R. S. Beroukim, T. L. Kruzic, A. L. Taylor, et al., "Progression of Aortic Dilatation in Children with a Functionally Normal Bicuspid Aortic Valve," American Journal of Cardiology, Vol. 98, No. 6, September 2006, pp. 828-830.

[4] G. Galanti, L. Stefani, L. Toncelli, et al., "Effects of Sport Activity in Athletes with Bicuspid Aortic Valve and Mild Aortic Regurgitation," British Journal of Sports Medicine, Vol. 44, No. 4, March 2010, pp. 275-279.

[5] P. W. Fedak, M. P. L. de Sa and S. Verna, "Vascular Matrix Remodeling in Patients with Bicuspid Aortic Valve Malformation Implication for Aortic Dilatation," Journal of Thoracic and Cardiovascular Surgery, Vol. 126, No. 3, September 2003, pp. 797-806.

[6] S. A. le Marie, X. Wang and J. A. Wilks, "Matrix Metalloproteinases in Ascending Aortic Aneurysm Bicuspid Versus Trilefted Aortic Valves," Journal of Surgical Re- 
search, Vol. 123, No. 1, 2005, pp. 40-48.

[7] E. Picano, "Stress Echocardiography," Expert Review of Cardiovascular Therapy, Vol. 2, No. 1, January 2004, pp. 77-88.

[8] C. S. Jr. Sidney, "ACC/AHA 2006 Guidelines for the Management of Patients with Valvular Heart Disease," Journal of the American College of Cardiology, Vol. 48, No. 3, May 2006, pp. e1-e148.

[9] J. H. Mitchell, W. Haskell, P. Snell, et al., "Task Force 8: Classification of Sport," Journal of the American College of Cardiology, Vol. 45, No. 8, April 2005, pp. 1364-1367.

[10] M. S. Fernandez, S. P. Sanders, P. Khairy, et al., "Morphology of Bicuspid Aortic Valve in Children and Adolescents," Journal of the American College of Cardiology, Vol. 44, No. 9, October 2004, pp. 1648-1651.

[11] M. D. Cheitilin, W. F. Armstrong and G. R. Aurigemma, "ACC/AHA/ASE 2003 Guideline Update for the Clinical Application of Echocardiography: A Report of the American College of Cardiology, American Heart Association Task Force on Practice Guidelines," Journal of the American Society of Echocardiography, Vol. 16, No. 10, October 2003, pp. 1091-1110.

[12] R. B. Devereux, "Detection of Left Ventricular Hypertrophy by M-Mode Echocardiography, Anatomic Validation, Standardization and Comparison to Other Methods," $\mathrm{Hy}$ pertension, Vol. 9, No. 2, February 1987, pp. 1119-1126.

[13] W. H. Gaasch, "Left Ventricular Radius to Wall Thickness Ratio," American Journal of Cardiology, Vol. 43,
No. 6, June 1979, pp. 1189-1194.

[14] R. L. Popp, "M-Mode Echocardiographic Assessment of Left Ventricular Function," American Journal of Cardiology, Vol. 49, No. 5, April 1982, pp. 1312-1318.

[15] R. Emmanuel, R. Withers and K. O’Brien, "Congenitally Bicuspid Aortic Valves, Clinicogenetic Study of 41 Families," British Regional Heart Study, Vol. 40, No. 3, December 1978, pp. 1420-1427.

[16] P. de Mozzi, U. G. Longo, G. Galanti and N. Maffulli, "Bicuspid Aortic Valve: A Literature Review and its Impact on Sport Acitivity," British Medical Bulletin, Vol. 85, No. 1, February 2008, pp. 63-85.

[17] C. Tribouilloy, F. Lévy, D. Rusinaru, et al., "Outcome after Aortic Valve Replacement for Low-Flow/LowGradient Aortic Stenosis without Contractile Reserve on Dobutamine Stress Echocardiography," Journal of the American College of Cardiology, Vol. 53, No. 20, May 2009, pp. 1865-1873.

[18] C. Bolcal, S. Doganci, O. Baysan, et al., "Evaluation of Left Ventricular Function after Aortic Valve Replacement in a Specific Young Male Patient Population with Pure Aortic Insufficiency or Stenosis: 5 Years Follow-up," Heart Surgical Forum, Vol. 10, No. 1, February 2007, pp. 57-63.

[19] M. L. Brown, H. V. Schaff, R. M. Suri, et al., "Index of Left Ventricular Dimensions Best Predict Survival after Aortic Valve Replacement in Patients with Aortic Valve Regurgitation," Annals of Thoracic Surgery, Vol. 87, No. 4, April 2009, pp. 1161-1169. 